\title{
Weight- perception in male career firefighters and its association with cardiovascular risk factors
}

\author{
Dorothee M Baur ${ }^{1,2}$, Costas A Christophi ${ }^{1,3}$, Antonios J Tsismenakis ${ }^{1,2,4}$, Sara A Jahnke ${ }^{5}$ and Stefanos N Kales ${ }^{1,2,6^{*}}$
}

\begin{abstract}
Background: The prevalence of obesity has reached epidemic proportions worldwide, and is also increasing among public safety professionals like firefighters who are expected to be fit and more active. The present study evaluates the associations among Body Mass Index (BMI), weight perception and cardiovascular risk factors in 768 male career firefighters from two Midwestern states in the United States.
\end{abstract}

Methods: A physical examination was performed and fasting blood samples were taken. Cardio-respiratory fitness (CRF) was determined from symptom- limited maximal treadmill exercise testing with electrocardiogram (ECG) monitoring and estimation of oxygen consumption (metabolic equivalents, METS) using the Bruce protocol. A health and lifestyle questionnaire was administered with standardized written instructions for completion. Self-reports of weight perception were extracted from responses to the completed multiple choice questionnaire. Baseline characteristics were described using the mean (standard deviation) for continuous variables and frequency for categorical variables. Group comparisons were calculated using analysis of variance (ANOVA). Linear models and logistic regression models were used to adjust for possible confounders. Logistic regression analyses were used to calculate the odds ratios of underestimating one's weight category.

Results: A high proportion of overweight and obese male career firefighters underestimate their weight categories (68\%). The risk of underestimating one's weight category increased by $24 \%$ with each additional unit of increasing BMI after adjustment for age and CRF. When divided into six groups based on combinations of measured BMI category and weight perception, there were significant differences among the groups for most cardiovascular risk factors. After adjustment for age and BMI, these differences remained statistically significant for CRF, amount of weekly exercise, prevalence of Metabolic Syndrome (MetSyn), body fat percentage and cholesterol measurements.

Conclusion: A high proportion of overweight and obese male career firefighters underestimate their measured BMI categories. As a result, they are unlikely to fully appreciate the negative health consequences of their excess weight. The results of this study emphasize the importance of objectively measuring BMI and then informing patients of their actual weight status and the associated disease risks.

Keywords: Weight perception, Cardio-respiratory fitness, Firefighters, Obesity, Cardiovascular-risk factors

\section{Background}

According to the World Health Organization (WHO), the prevalence of obesity in the US has reached $44 \%$ in males between 30 and 100 years of age [1]. Higher Body Mass Index (BMI) has been associated with increased risk of cardiovascular disease (CVD) and certain types of

\footnotetext{
* Correspondence: skales@challiance.org

'Department of Environmental Health, Environmental and Occupational Medicine and Epidemiology (EOME), Harvard School of Public Health, Boston, MA, USA

${ }^{2}$ The Cambridge Health Alliance, Harvard Medical School, Employee Health and Industrial Medicine, Cambridge, MA, USA

Full list of author information is available at the end of the article
}

cancer $[2,3]$. On the other hand, weight loss has been shown to decrease the risks associated with excess weight and obesity. Even a small amount of weight loss has positive effects on cardiovascular risk factors [4].

Perception of one's weight status as non-ideal is usually the basis for any subsequent decision to change one's weight. Accordingly, there is a strong association between perception of weight and effective weight control in adults [5]. Therefore, accurate awareness of weight status is an increasingly important adjunct to achieving successful weight loss. Unfortunately, recent evidence emphasizes that the rapid societal increase in overweight and obesity

\section{Biomed Central}


has been accompanied by fewer overweight people correctly perceiving themselves as belonging to the overweight category [6]. One recent study found that twothirds of already obese individuals did not recognize their obesity. Moreover, despite their already excessive weights they believed they were at low risk of developing obesity [7].

In parallel, recent research has found that obesity in the United States (US) fire service has reached epidemic levels [8-11]. In a prospective evaluation, obesity prevalence in this occupation increased significantly over time [8]. Moreover, a recent population-based investigation of career and volunteer firefighters used BMI, waist circumference and body fat measures to validate prevalence estimates done using BMI with the latter measures of abdominal obesity and adiposity (fat mass) [10]. This investigation proved that the high obesity prevalence found among firefighters using BMI was not due to misclassification of increased muscle mass as adiposity (fat mass), which was contrary to popular belief in the fire service. In fact, obesity was even more prevalent when assessed by body fat rather than BMI, while the misclassification of muscular firefighters as obese by BMI was infrequent [10]. Furthermore we have described that obesity defined using BMI measures is associated with CVD risk factor clustering [8,9], lower cardiorespiratory fitness (CRF) $[9,12,13]$ as well as higher risk of job disability in firefighters [14].

In agreement with the above results, several general population studies have recently reported an increase in overweight and obesity over time. All but one [15] also described a parallel increase in misperceptions of excess weight, expressed as failing to recognize oneself as being overweight or obese $[6,16,17]$.

Recently, body weight misperception has been shown to be associated with gender, health beliefs, ethnicity and socioeconomic status $[5,7,18,19]$. However, to the best of our knowledge, no study has previously described weight perception and its association with cardiovascular risk factors, CRF and body composition in firefighters or even in the general population per se. The purposes of this study were to describe and quantify the discrepancy between weight perception and actual BMI in a high risk public safety profession; and secondarily, to compare CVD risk factors across categories of measured BMI and perceived weight status.

\section{Methods}

\section{Study population}

Male career firefighters, 18 years of age and older, were recruited from fire departments in two Midwestern states. Inclusion criteria were completing a maximal exercise test during the course of a fire department- sponsored medical examination, and working full duty without any work restrictions at the time of the examination. Excluded subjects failed to meet one or more of the above criteria or had undergone the index exercise tests for the evaluation of symptoms, retirement pensions, disability and/or exit examinations. The study was approved by the IRB of Harvard School of Public Health and local IRBs as appropriate. All participants signed an informed consent.

\section{Assessment of cardiovascular risk factors}

Height was measured in the standing position with a clinic stadiometer. Body weight was measured with bare feet and in light clothes on a calibrated scale. BMI was calculated as the weight in kilograms divided by the square of height in meters. Body fat percentage (body fat\%) was either estimated by the use of a Bioelectrical Impedance Analyzer (BIA) or with skin fold measures depending on the protocol of the respective fire department. Blood pressure was measured using an appropriately sized cuff with the subject in the seated position. Resting pulse and blood pressure were obtained from the physical examination. Fasting venous blood samples were analyzed for total cholesterol (total chol), HDL-cholesterol (HDL-chol), LDL-cholesterol (LDL-chol), the ratio between total- and HDL chol (Tchol/HDL), c- reactive protein and glucose using standardized methods.

\section{Cardio-respiratory fitness}

CRF was measured using symptom- limited maximal treadmill exercise testing with ECG monitoring and estimation of oxygen consumption (metabolic equivalents (METS)) according to the Bruce protocol [20]. The participants were encouraged to continue exercise until volitional exhaustion; even after exceeding $85 \%$ of their maximum predicted heart rate (maximal predicted heart rate is defined as 220 minus age). The cohort achieved an average of $97.9 \%$ (SD 6.6) of maximal age-predicted heart rate on these tests. During the exercise test CRF was determined from the maximum or peak METS achieved at peak exercise. Heart rate recovery at one minute (HHR1) was calculated as peak heart rate minus heart rate at one minute into recovery following the test.

\section{Definition of the metabolic syndrome (MetSyn)}

The prevalence of MetSyn among the study population was determined using modified criteria from the Joint Scientific Statement [21,22]. If three or more of the following five risk factors were present then the participant was categorized as having MetSyn: abdominal obesity- modified here to $\mathrm{BMI} \geq 30$; hypertriglyceridemia $(\geq 150 \mathrm{mg} / \mathrm{dL})$; reduced HDL-chol $<40 \mathrm{mg} / \mathrm{dL}$; elevated blood pressure (systolic $\geq 130$ and/or diastolic $\geq 85 \mathrm{mmHg}$ ) and/or antihypertensive drug treatment; or hyperglycemia (blood glucose $\geq 100 \mathrm{mg} / \mathrm{dL}$ ) [21]. 


\section{Assessment of weight perception and weekly exercise}

Self-reports of weight perception and weekly exercise were extracted from responses to a health and lifestyle questionnaire as previously described from our group [12]. Consented study participants were given standardized written instructions for completing the multiple choice survey regarding eating, health, exercise, sleep, and work habits as honestly and as best as they could. They were also informed that the completed questionnaires would be confidential and would not become part of their fire department or medical record. To assess weekly exercise, the following question was asked: "Most weeks, I exercise .... (include home/work/gym \& elsewhere)" [possible answers: one day or less, 2-4 days, 5 days or more]. To assess weight perception, answers to the following questions were selected. (1) "I think my body weight is... "[possible answers: underweight (skinny); healthy/normal or muscular; overweight; obese (fat)]. (2) "In the next year, I want my body weight to go ... "[possible answer: Down a lot ( $>10$ pounds); Down a little (5-10 pounds); Not changed ( $<5$ pounds); Up a little (5-10 pounds); Up a lot ( $>10$ pounds)].

The combination of objectively measured BMI groups and the self-perceived weight categories gave rise to 12 groups (BMI category/self-perception weight category). The following groups were not included in the analyses due to very small numbers: [overweight BMI/underweight (skinny); obese $\mathrm{BMI} /$ underweight (skinny); normal BMI/obese $(\mathrm{n}=0)]$ and [normal BMI/underweight (skinny); normal BMI/overweight; overweight $\mathrm{BMI} /$ obese (fat) $(\mathrm{n} \leq 9)]$.

\section{Statistical analysis}

Baseline characteristics were described using the mean (SD) in the case of quantitative variables and the frequency in case of categorical variables. Group comparisons were calculated using ANOVA. Linear regression models were used to evaluate the associations among the six BMI/weight perception categories (independent variable) and various cardiovascular risk factors (dependent variables) while adjusting for other co-variables. Logistic regression analyses were used to calculate odds ratios (95\% CI) for underestimating one's weight category (dependent variable) as a function of BMI as a continuous independent variable. Analyses were performed using SAS 9.2 (SAS Institute Inc., Cary, NC, USA). All tests presented are two-sided and a p-value $<0.05$ is considered significant.

\section{Results}

The cohort's $(n=768)$ baseline characteristics are summarized in Table 1. The mean BMI and body fat were both close to respective obesity cutoff criteria: 29.4 (4.4) versus $30 \mathrm{~kg} / \mathrm{m}^{2}$ and $23.6 \%$ versus $25 \%$. The prevalence
Table 1 Baseline characteristics $(n=768)$

\begin{tabular}{ll}
\hline Age mean (SD) & $37.6(8.5)$ \\
\hline BMI mean (SD) & $29.4(4.4)$ \\
\hline CRF (maximal METS) mean (SD) & $12.8(1.6)$ \\
\hline Body fat\% mean (SD) & $23.6(6.6)$ in $\mathrm{n}=232$ \\
\hline Blood glucose mean (SD) & $94.0(21.0)$ \\
\hline Resting systolic BP mean (SD) & $122.6(12.6)$ \\
\hline Resting diastolic BP mean (SD) & $79.9(8.0)$ \\
\hline Resting heart rate mean (SD) & $69.5(11.4)$ \\
\hline HDL-chol mean (SD) & $45.3(11.2)$ \\
\hline LDL-chol mean (SD) & $120.7(34.1)$ \\
\hline Total- chol mean (SD) & $195.2(38.7)$ \\
\hline TChol/HDL ratio mean (SD) & $4.6(1.5)$ \\
\hline Heart rate recovery at one minute mean (SD) & $33.1(13.5)$ \\
\hline C-reactive protein mean (SD) & $2.2(3.7)$ in $\mathrm{n}=389$ \\
\hline
\end{tabular}

BMI (Body Mass Index), CRF (Cardio-Respiratory Fitness), BP (Blood Pressure), chol (Cholesterol), SD (Standard Deviation).

of underestimating one's weight category furthermore increased with age ( $p$-value $<0.0001)$. Figure 1 illustrates firefighters' perceptions of their weight within each measured BMI category. In the measured normal BMI category $(\geq 18.5$ and $<25)$, $89 \%$ of the study participants perceived themselves correctly as "healthy/normal or muscular". In the overweight BMI category $(25 \leq \mathrm{BMI}$ $<30$ ) only $32.4 \%$ rated themselves correctly as overweight. Furthermore in the obese BMI category (BMI $\geq$ 30) only $8.2 \%$ of firefighters perceived themselves correctly as obese (Figure 1). Moreover, with every one unit increment in the BMI, the odds of underestimating one's BMI measured category was increased by $24 \%$ [OR = 1.241 (95\% CI 1.176-1.308)] after adjustment for age and CRF (Table 2). However, if we examined only obese subjects $(\mathrm{BMI} \geq 30$ ), as obesity increased, the probability of correctly perceiving one's measured weight category actually increased as BMI increased (Figure 2).

Cardiovascular risk factors are described and compared among the six different $\mathrm{BMI} /$ weight perception groups in Table 3. With and without adjustment for age, differences were statistically significant among the groups for CRF, amount of weekly exercise, MetSyn prevalence, resting systolic and diastolic blood pressure, HDL-, LDLand total chol as well as for the ratio between total- and HDL chol, body fat $\%$, c- reactive protein and glucose (Table 3). If additionally adjusted for BMI, the differences remained statistically significant for CRF, amount of weekly exercise, MetSyn, HDL- LDL- and total chol as well as Tchol/HDL and body fat $\%$.

Among the three groups where measured BMI category is normal or overweight, the following parameters were significantly different: CRF, amount of weekly exercise, MetSyn, HRR1, HDL-, LDL- and total chol, as well as the Tchol/HDL, body fat $\%$ and blood glucose. After 


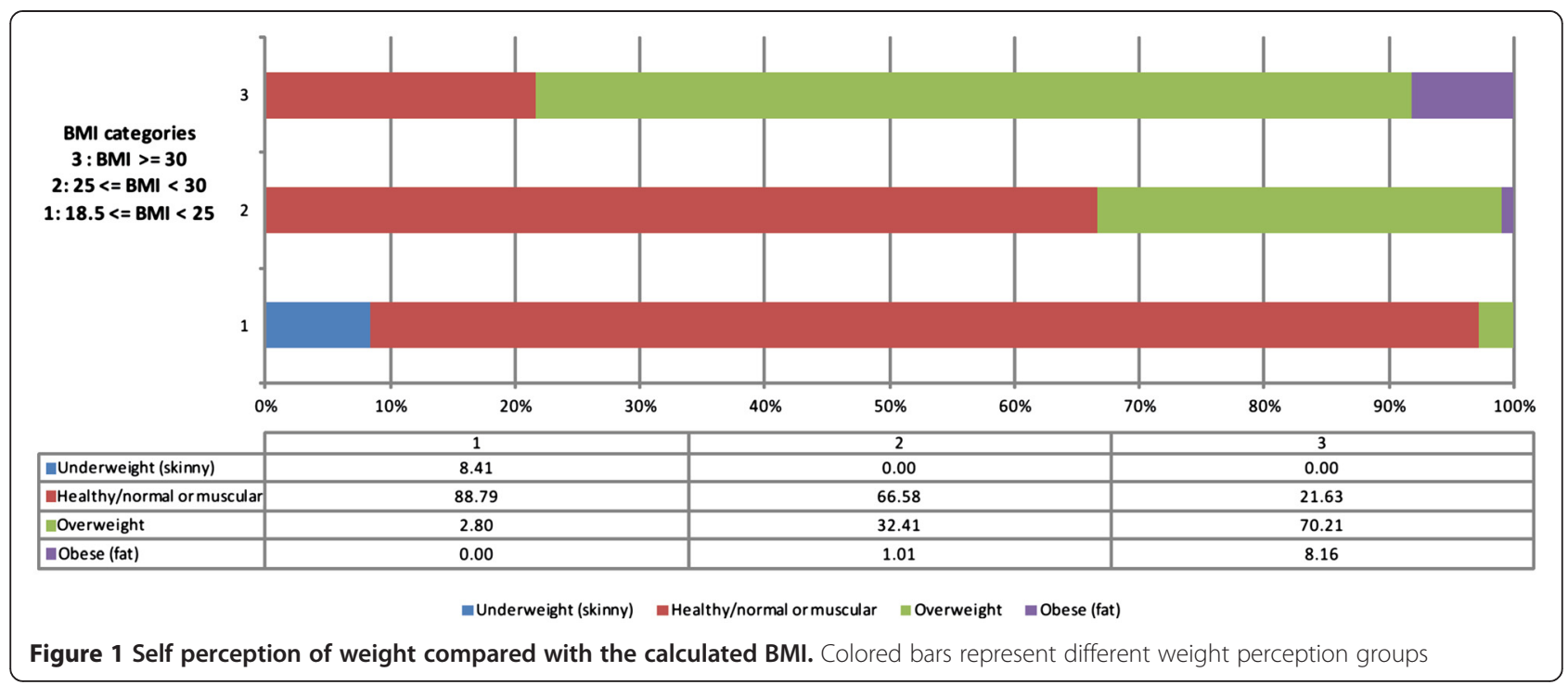

adjustment for age and BMI the following parameters were significantly different: CRF, amount of weekly exercise, HDL- and total chol, as well as the Tchol/HDL and body fat\%.

In comparison when analyses were limited to only those groups where measured BMI was in the obese category (but weight perception was normal, overweight or obese), the analysis showed significant differences for CRF, body fat\%, Tchol/HDL and HDL. After adjustment for age and BMI, only the difference for total chol remained significant.

Additionally the three groups of obese participants answered differently when asked about desired weight change in the next year based on their self-perceptions of their weight status. In the group that perceived themselves of normal weight $34.4 \%$ wished to reduce their weight more than 10 pounds compared to $69 \%$ among those perceiving themselves as overweight and $82.6 \%$ in the group that perceived themselves correctly as obese (p-value overall $<0.0001$ ).

\section{Discussion}

We found that a high proportion of overweight and obese male career firefighters underestimate their weight group (68\%). This is consistent with previously reported findings in the literature [23]. We also found the risk of underestimating one's weight category increased by $24 \%$

Table 2 Odds Ratios of underestimating one's BMI category as a function of increasing BMI (continuous)

\begin{tabular}{llll}
\hline & Model 1 & Model 2 & Model 3 \\
\hline OR (95\% Cl) & $1.201(1.146-1.258)$ & $1.210(1.154-1.269)$ & $1.241(1.176-1.308)$
\end{tabular}

Model 1: unadjusted; Model 2: adjusted for age; Model 3: adjusted for age and CRF (maximal METS).

BMI (Body Mass Index), CRF (Cardio-Respiratory Fitness), METS (Metabolic Equivalent), OR (Odds Ratio), $\mathrm{Cl}$ (Confidence Interval). with each additional unit of BMI after adjustment for age and CRF. These findings are also consistent with other studies $[6,16,17]$.

Novel findings from this study relate to cardiovascular risk factors as a function of weight perception. We found that risk parameters varied significantly not only with measured BMI, but also with weight perception. Among those who are overweight or obese, CVD risk showed worsening trends as the BMI increased and weight perception became more accurate. This finding seems to be explained by further increases in BMI and body fat that are found among overweight and obese persons who correctly perceive themselves as overweight or obese, rather than normal or muscular. In fact, obese firefighters who perceived themselves as obese were close to morbidly obese on average (BMI $>39)$. An alternate view (and not mutually exclusive one) is that overweight and obese subjects who view themselves as "normal/healthy or muscular" are actually more active, have higher CRF and better CVD risk profiles than obese subjects who perceive themselves as heavier. However, in the present study, while this was true, overweight and obese participants who perceive themselves as normal/ muscular still have worse CVD risk parameters than colleagues whose measured BMI is normal. Thus, any differences based on perception appear to be accounted for by differences in the degree of adiposity as measured by BMI and body fat.

Correctly perceiving one's BMI category does not only imply knowing one's weight relative to their height. It also seems to be dependent on social standards and peer groups. If most of the peers one compares himself to are overweight or obese, there is a danger of perceiving overweight as "normal" or "standard size" [23]. In our cohort $86.1 \%$ of firefighters were overweight and obese, 


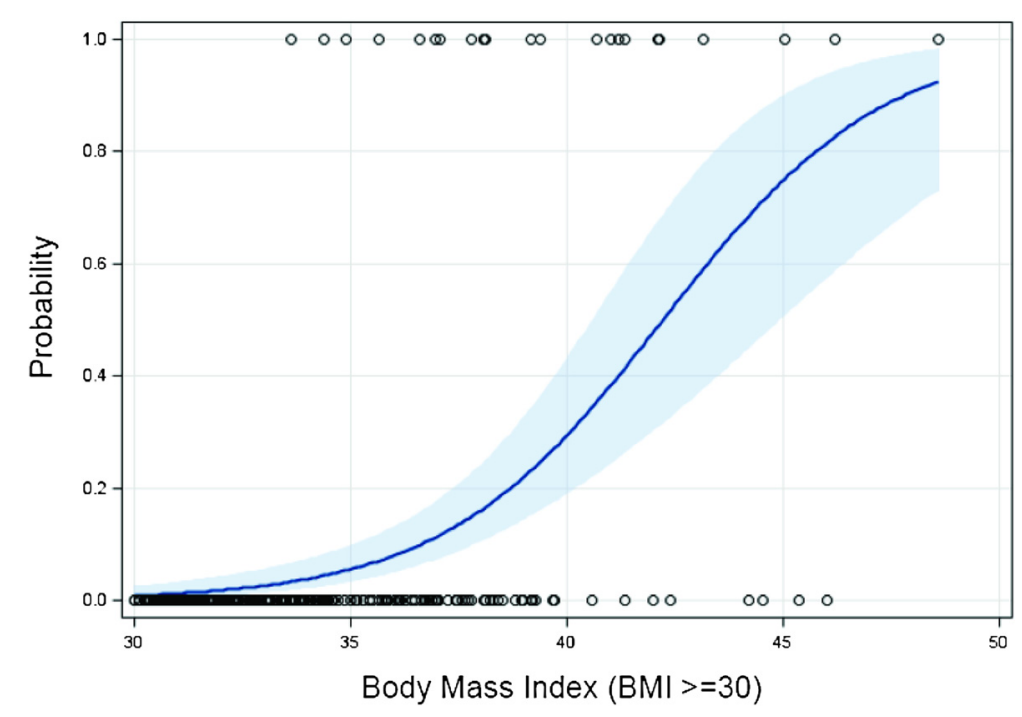

Figure 2 Prediction probabilities and $95 \%$ prediction limits of correctly perceiving weight group as a function of BMI among obese participants.

so it is quite likely that shifts in standards of weight perceptions are occurring in the fire service. During the past decade the desired weight of Americans has increased alongside the increase of obesity. This is suggestive that at some point the assessment of body weight is made in relation to distribution of weight in the general population [24].

An additional interesting finding regarded risk factor clustering. Our study showed that the MetSyn prevalence was highly dependent on both measured and perceived weight categories. This was especially true in those with a BMI measured in the obese category. The prevalence of MetSyn was quite high among obese firefighters and increased from $54.1 \%$ (self-perceived normal/healthy/muscular) up to $78.3 \%$ (self-perceived obese). Therefore our data may suggest that especially if subjects are obese but relatively healthy they may be more likely to underestimate their weight category as well as their weight-related health risks. Recognizing this might provide an opportunity for increasing and emphasizing education concerning healthy weight and weight maintenance strategies [25]. While the less obese subjects who perceive themselves as healthy are in fact healthier than even heavier co-workers, they are clearly much less healthy than leaner co-workers on multiple objective measures.

\section{Strengths of the present study}

A particular strength of the present study was the large sample size which allowed for adequate power and adjustment for confounders. BMI was calculated from measured weight and height in bare feet and light clothes during medical examinations which avoided self- reporting biases towards lower weights and taller heights and other random misclassification. We also were able to compare the associations of perceived weight category on objectively measured markers of CVD risk, which permitted us to characterize the health status of the various subgroups. Moreover, our study distinguished between overweight and obese. When most overweight or obese people perceive themselves as overweight, they fail to appreciate the extent of how far they are from their healthy weights [5]. Finally because of the very homogenous study group there was no need to adjust for socioeconomic status (education, income, occupation).

\section{Limitations of the present study}

Our study does have some modest limitations, including the cross-sectional design. Because of previously described gender differences in weight perception and the very small number of female career firefighters, we limited our investigation to male firefighters. Furthermore, although we had very complete data overall, in the case of body fat $\%$ and c-reactive protein measurements, some firefighters were examined before these items were added to their departments' medical exam protocols. Therefore, we lacked complete data for these two variables. Finally, with regard to body composition and MetSyn determination, we lacked measures of waist circumference. However, BMI has been shown to be accurate measure of adiposity in firefighters [10].

\section{Conclusion}

We found that a high proportion of overweight and obese male career firefighters underestimate their measured BMI categories. In fact, the risk of underestimating 
Table 3 Cardiovascular Risk Factors as a function of Body Mass Index and weight perception categories

\begin{tabular}{|c|c|c|c|c|c|c|c|c|c|}
\hline n & 95 & 263 & 128 & 61 & 198 & 23 & p-value ${ }^{2}$ & $p$-value ${ }^{3}$ & p-value ${ }^{4}$ \\
\hline BMI category & normal & overweight & overweight & obese & obese & obese & $\mathrm{n} / \mathrm{a}$ & $\mathrm{n} / \mathrm{a}$ & $\mathrm{n} / \mathrm{a}$ \\
\hline Self reported weight category ${ }^{\prime}$ & healthy/nl & healthy/nl & overweight & healthy/nl & overweight & obese & $\mathrm{n} / \mathrm{a}$ & $n / a$ & $\mathrm{n} / \mathrm{a}$ \\
\hline weight perception & correct & underestim & correct & underestim & underestim & correct & $\mathrm{n} / \mathrm{a}$ & $\mathrm{n} / \mathrm{a}$ & $\mathrm{n} / \mathrm{a}$ \\
\hline Age mean (SD) & $35.9(9.9)$ & $36.0(8.2)$ & $39.4(8.5)$ & $36.4(7.8)$ & $39.3(8.0)$ & $39.3(6.9)$ & $<0.0001$ & $n / a$ & $\mathrm{n} / \mathrm{a}$ \\
\hline BMl mean (SD) & $23.7(1.0)$ & $27.2(1.3)$ & $28.3(1.4)$ & $32.5(2.3)$ & $33.8(3.1)$ & $39.6(3.8)$ & $<0.0001$ & $<0.0001$ & $\mathrm{n} / \mathrm{a}$ \\
\hline CRF (maximal METS) mean (SD) & $13.7(1.6)$ & $13.4(1.2)$ & $12.6(1.3)$ & $12.6(1.3)$ & $11.8(1.6)$ & $10.8(1.6)$ & $<0.0001$ & $<0.0001$ & 0.0052 \\
\hline $\begin{array}{l}\text { Weekly exercise (days/week) } \\
\text { (one day or less) n (\%) }\end{array}$ & $15(16.0)$ & $18(6.9)$ & $30(23.4)$ & $5(8.2)$ & $44(22.5)$ & $10(43.5)$ & $<0.0001$ & 0.0010 & $<0.0001$ \\
\hline Weekly exercise (2-4 days) n (\%) & $53(56.4)$ & $157(60.2)$ & $84(65.6)$ & $36(59.0)$ & $130(66.3)$ & $13(56.5)$ & & & \\
\hline Weekly exercise ( 5 days or more) $\mathrm{n}(\%)$ & $26(27.7)$ & $86(33.0)$ & $14(10.9)$ & $20(32.8)$ & $22(11.2)$ & $0(0.0)$ & & & \\
\hline MetSyn n(\%) & $4(4.2)$ & $20(7.6)$ & $20(15.6)$ & $33(54.1)$ & $118(59.6)$ & $18(78.3)$ & $<0.0001$ & $<0.0001$ & $<0.0001$ \\
\hline HRR1 mean (SD) & $36.5(14.4)$ & $33.8(14.0)$ & $31.3(13.7)$ & $32.3(11.7)$ & $32.2(12.9)$ & $30.5(10.8)$ & 0.0702 & 0.2069 & 0.5954 \\
\hline RSBP mean (SD) & $118.8(12.3)$ & $121.1(12.0)$ & $121.4(12.4)$ & $125.6(14.6)$ & $125.8(12.5)$ & $125.5(10.4)$ & $<0.0001$ & $<0.0001$ & 0.6621 \\
\hline RDBP mean (SD) & $76.7(8.0)$ & $78.3(7.6)$ & $79.5(7.4)$ & $82.0(8.2)$ & $83.0(7.8)$ & $81.5(5.6)$ & $<0.0001$ & $<0.0001$ & 0.1229 \\
\hline HDL- chol mean (SD) & $50.4(11.0)$ & $48.7(11.3)$ & $43.2(9.6)$ & $43.3(10.0)$ & $40.8(10.1)$ & $40.8(11.4)$ & $<0.0001$ & $<0.0001$ & 0.0004 \\
\hline LDL- chol mean (SD) & $109.4(34.0)$ & $117.7(34.0)$ & $129.3(33.8)$ & $116.8(25.1)$ & $125.9(34.3)$ & $121.8(41.5)$ & $<0.0001$ & 0.0023 & 0.0054 \\
\hline TChol mean (SD) & $181.7(37.1)$ & $191.9(38.1)$ & $203.7(43.6)$ & $191.0(29.1)$ & $200.3(36.2)$ & $211.0(46.0)$ & $<0.0001$ & 0.0010 & 0.0020 \\
\hline TChol/HDL ratio mean (SD) & $3.8(1.3)$ & $4.2(1.4)$ & $4.9(1.5)$ & $4.6(1.3)$ & $5.1(1.4)$ & $5.5(1.7)$ & $<0.0001$ & $<0.0001$ & 0.0002 \\
\hline Body fat(\%) mean (SD) & $16.0(6.8) n=32$ & $19.8(4.0) n=72$ & $23.8(3.4) n=36$ & $27.4(4.4) n=20$ & $29.1(3.6) n=64$ & $32.5(4.4) n=8$ & $<0.0001$ & $<0.0001$ & 0.0055 \\
\hline c-reactive protein mean (SD) & $1.5(2.6) n=58$ & $1.8(3.9) n=136$ & $2.4(6.0) n=48$ & $2.0(2.3) n=35$ & $3.1(2.6) n=102$ & $3.4(3.6) n=10$ & 0.0331 & 0.0336 & 0.7612 \\
\hline Blood glucose mean (SD) & $87.2(12.2)$ & $92.4(20.8)$ & $94.8(13.7)$ & $94.8(21.8)$ & $97.7(26.9)$ & $101.7(19.1)$ & 0.0009 & 0.0105 & 0.8976 \\
\hline
\end{tabular}

\section{${ }^{1}$ Healthy/normal or muscular; Overweight; Obese (fat).}

${ }^{2}$ Crude; ${ }^{3}$ age adjusted; ${ }^{4}$ adjusted for age and BMI.
$B M I$ (Body Mass Index), CRF (Cardio-Respiratory Fitness), METS (Metabolic Equivalent), MetSyn (Metabolic Syndrome), HRR1 (Heart Rate Recovery at one minute into recovery), RSBP (Resting Systolic Blood Pressure), RDBP (Resting Diastolic Blood Pressure), TChol/HDL (Ratio between total cholesterol and HDL).
(Rody 
one's BMI category increased by $24 \%$ with each additional unit of measured BMI after adjustment for age and CRF. Furthermore when divided into six groups based on combination of measured BMI category and weight self-perception, there were significant differences among the groups for most cardiovascular risk factors which remained statistically significant for CRF, amount of weekly exercise, prevalence of MetSyn, body fat percentage and cholesterol measurements after adjustment for age and BMI.

The results of this study emphasize the importance of objectively determining BMI and then informing patients of their actual weight status and the associated disease risks. This is of particular importance in public safety occupational settings like firefighting where the public depends on workers to be healthy and fit for duty.

\section{Competing interests}

The other authors report no conflict of interest.

\section{Authors' contributions}

DMB contributed to the study design, performed statistical analyses, wrote and edited the manuscript; CAC performed statistical analyses and edited manuscript; AJT contributed to the study design, drafted and edited manuscript; SAJ participated in study recruitment and design and edited manuscript; SNK planned and designed the study and edited manuscript. All authors read and approved the final manuscript.

\section{Acknowledgements \\ The authors would like to thank all of the participating firefighters and Fire Departments; the staff and clinical leadership of the clinics who examined the firefighters; Ms. Brianne Tuley, Dr. Lilly Ramphal and the late Dr. William Patterson for their contributions to the underlying longitudinal study. This investigation was supported by the Federal Emergency Management Agency (FEMA) Assistance to Firefighters Grant (AFG) program's awards EMW-2006-FP-01493 (PI: Dr. S.N. Kales), EMW-2009-FP-00835 (PI: Dr. S.N. Kales).}

\section{Author details}

${ }^{1}$ Department of Environmental Health, Environmental and Occupational Medicine and Epidemiology (EOME), Harvard School of Public Health, Boston, MA, USA. ${ }^{2}$ The Cambridge Health Alliance, Harvard Medical School, Employee Health and Industrial Medicine, Cambridge, MA, USA. ${ }^{3}$ Cyprus International Institute for Environmental and Public Health in association with Harvard School of Public Health, Cyprus University of Technology, Limassol, Cyprus. ${ }^{4}$ Boston University School of Medicine, Boston, MA 02118, USA. ${ }^{5}$ The Institute for Biobehavioral Health Research, National Development and Research Institutes, Leawood, KS, USA. ${ }^{6}$ The Cambridge Health Alliance, 1493 Cambridge Street. Macht 427, Cambridge, MA 02139, USA

\section{Disclosure}

S.N. Kales has served as expert witness in legal cases involving firefighters and is working under contract to update a heart disease manual for the International Association of Fire Fighters (IAFF).

Received: 23 December 2011 Accepted: 25 June 2012 Published: 25 June 2012

\section{References}

1. WHO Global Infobase:.; ; https://apps.who.int/infobase/Comparisons.aspx?l= \&NodeVal=WGIE BMI 5 cd.0704\&DO=1\&DDLReg=ALL\&DDLSex= 1\&DDLAgeGrp=15-100\&DDLYear=2010\&DDLMethod=INTMDCTM\& DDLCateNum $=6 \&$ TxtBxCtmNum $=20 \% 2 c 35 \% 2 c 50 \% 2 c 65 \% 2 c 80 \& C B L C 1=$ $\mathrm{ON} \& C B L C 3=\mathrm{ON} \& C B L C 4=\mathrm{ON} \& C B L C 6=\mathrm{ON} \& C B L C 8=\mathrm{ON} \& C B L C 10=$ ON\&DDLMapsize $=800 \times 480 \& D D L M a p L a b e l s=$ none\&DDLTmpRangBK= 0\&DDLTmpColor $=-3342388$
2. Flegal KM, Graubard BI, Williamson DF, Gail MH: Cause-specific excess deaths associated with underweight, overweight, and obesity. JAMA 2007, 298(17):2028-2037

3. de Gonzalez A, Hartge P, Cerhan JR, Flint AJ, Hannan L, MacInnis RJ, Moore SC, Tobias GS, Anton-Culver H, Freeman LB, et al: Body-mass index and mortality among 1.46 million white adults. N Engl J Med 2010, 363(23):2211-2219.

4. Elmer PJ, Obarzanek E, Vollmer WM, Simons-Morton D, Stevens VJ, Young DR, Lin PH, Champagne C, Harsha DW, Svetkey LP, et al: Effects of comprehensive lifestyle modification on diet, weight, physical fitness, and blood pressure control: 18-month results of a randomized trial. Ann Intern Med 2006, 144(7):485-495.

5. Chang W, Christakis NA: Self-perception of weight appropriateness in the United States. Am J Prev Med 2003, 24(4):332-339.

6. Johnson-Taylor WL, Fisher RA, Hubbard VS, Starke-Reed P, Eggers PS: The change in weight perception of weight status among the overweight: comparison of NHANES III (1988-1994) and 1999-2004 NHANES. Int J Behav Nutr Phys Act 2008, 5:9.

7. Powell TM, de Lemos JA, Banks K, Ayers CR, Rohatgi A, Khera A, McGuire DK, Berry JD, Albert MA, Vega GL, et al: Body size misperception: a novel determinant in the obesity epidemic. Arch Intern Med 2010, 170(18):1695-1697.

8. Soteriades ES, Hauser R, Kawachi I, Liarokapis D, Christiani DC, Kales SN: Obesity and cardiovascular disease risk factors in firefighters: a prospective cohort study. Obes Res 2005, 13(10):1756-1763.

9. Tsismenakis AJ, Christophi CA, Burress JW, Kinney AM, Kim M, Kales SN: The obesity epidemic and future emergency responders. Obesity (Silver Spring) 2009, 17(8):1648-1650.

10. Poston WS, Haddock CK, Jahnke SA, Jitnarin N, Tuley BC, Kales SN: The prevalence of overweight, obesity, and substandard fitness in a population-based firefighter cohort. J Occup Environ Med 2011, 53(3):266-273.

11. Soteriades ES, Smith DL, Tsismenakis AJ, Baur DM, Kales SN: Cardiovascular Disease in US Firefighters: A Systematic Review. Cardiol Rev 2011, 19(4):202-215

12. Durand G, Tsismenakis AJ, Jahnke SA, Baur DM, Christophi CA, Kales SN: Firefighters' physical activity: relation to fitness and cardiovascular disease risk. Med Sci Sports Exerc 2011 Sept, 43(9):1752-9.

13. Baur DM, Christophi CA, Tsismenakis AJ: Cook EF. Kales SN: Cardiorespiratory Fitness Predicts Cardiovascular Risk Profiles in Career Firefighters. J Occup Environ Med; 2011

14. Soteriades ES, Hauser R, Kawachi I, Christiani DC, Kales SN: Obesity and risk of job disability in male firefighters. Occup Med (Lond) 2008, 58(4):245-250.

15. Marques-Vidal P, Melich-Cerveira J, Marcelino G, Paccaud F: High- and persistent- body-weight misperception levels in overweight and obese Swiss adults, 1997-2007. Int J Obes (Lond) 2011 Dec, 35(12):1549-50. doi:10.1038/ijo.2010.285. Epub 2011 Jan 25. No abstract available.

16. Johnson F, Cooke L, Croker H, Wardle J: Changing perceptions of weight in Great Britain: comparison of two population surveys. BMJ 2008, 337: a494.

17. Salcedo V, Gutierrez-Fisac JL, Guallar-Castillon P, Rodriguez-Artalejo F: Trends in overweight and misperceived overweight in Spain from 1987 to 2007. Int J Obes (Lond) 2010, 34(12):1759-1765.

18. Bhanji S, Khuwaja AK, Siddiqui F, Azam I, Kazmi K: Underestimation of weight and its associated factors among overweight and obese adults in Pakistan: a cross sectional study. BMC Publ Health 2011, 11:363.

19. Duncan DT, Wolin KY, Scharoun-Lee M, Ding EL, Warner ET, Bennett GG: Does perception equal reality? Weight misperception in relation to weightrelated attitudes and behaviors among overweight and obese US adults. Int J Behav Nutr Phys Act 2011, 8:20.

20. Bruce RA: Exercise testing of patients with coronary heart disease. Principles and normal standards for evaluation. Ann Clin Res 1971 3(6):323-332.

21. Alberti KG, Eckel RH, Grundy SM, Zimmet PZ, Cleeman Jl, Donato KA, Fruchart JC, James WP, Loria CM, Smith SC Jr: Harmonizing the metabolic syndrome: a joint interim statement of the International Diabetes Federation Task Force on Epidemiology and Prevention; National Heart, Lung, and Blood Institute; American Heart Association; World Heart Federation; International Atherosclerosis Society; and International Association for the Study of Obesity. Circulation 2009, 120(16):1640-1645. 
22. Baur DM: Christophi CA. Kales SN: Metabolic Syndrome is Inversely Related to Cardio-Respiratory Fitness in Male Career Firefighters. J Strength Cond Res; 2011.

23. Gregory CO, Blanck HM, Gillespie C, Maynard LM, Serdula MK: Health perceptions and demographic characteristics associated with underassessment of body weight. Obesity (Silver Spring) 2008, 16(5):979-986.

24. Maynard LM, Serdula MK, Galuska DA, Gillespie C, Mokdad AH: Secular trends in desired weight of adults. Int J Obes (Lond) 2006, 30(9):1375-1381.

25. Godino JG, Lepore SJ, Rassnick S: Relation of misperception of healthy weight to obesity in urban black men. Obesity (Silver Spring) 2010, 18(7):1318-1322.

doi:10.1186/1471-2458-12-480

Cite this article as: Baur et al:: Weight- perception in male career firefighters and its association with cardiovascular risk factors. $B M C$ Public Health 2012 12:480.

\section{Submit your next manuscript to BioMed Central and take full advantage of:}

- Convenient online submission

- Thorough peer review

- No space constraints or color figure charges

- Immediate publication on acceptance

- Inclusion in PubMed, CAS, Scopus and Google Scholar

- Research which is freely available for redistribution 